\title{
Stable expression of Leptospira interrogans antigens in auxotrophic Mycobacterium bovis BCG
}

\author{
FABIANA K. SEIXAS ${ }^{1}$; SIBELE BORSUK ${ }^{1}$; MICHEL Q. FAGUNDES ${ }^{1}$; \\ DAIANE D. HARTWIG ${ }^{1}$; ÉVERTON F. DA SILVA ${ }^{1}$; GUSTAVO M. CERQUEIRA ${ }^{1}$ \\ and ODIR A. DELLAGOSTIN ${ }^{1, *}$
}

\footnotetext{
${ }^{1}$ Centro de Biotecnologia. Universidade Federal de Pelotas, Campus Universitário, 96010-900, Pelotas - RS, Brazil.
}

\begin{abstract}
Mycobacterium bovis BCG has been proposed as an effective live vector for multivalent vaccines. The development of mycobacterial genetic systems to express foreign antigens and the adjuvanticity of BCG are the basis for the potential use of this attenuated mycobacterium as a recombinant vaccine vector. Stable plasmid vectors without antibiotic resistance markers are needed for heterologous antigen expression in BCG. Our group recently described the construction of a BCG expression system using auxotrophic complementation as a selectable marker. In this work, LipL32 and LigAni antigens of Leptospira interrogans were cloned and expressed in M. bovis BCG Pasteur and in the auxotrophic M. bovis BCG $\Delta l e \mathrm{u} D$ strains under the control of the $M$. leprae $18 \mathrm{kDa}$ promoter. Stability of the plasmids during in vitro growth and after inoculation of the recombinant BCG strains in hamsters was compared. The auxotrophic complementation system was highly stable, even during in vivo growth, as the selective pressure was maintained, whereas the conventional vector was unstable in the absence of selective pressure. These results confirm the usefulness of the new expression system, which represents a huge improvement over previously described expression systems for the development of BCG into an effective vaccine vector.
\end{abstract}

Key terms: recombinant BCG, Auxotrophic complementation, foreign antigens

\section{INTRODUCTION}

Mycobacterium bovis BCG is an attractive candidate for the development of live recombinant vaccines. BCG offers unique advantages as a vaccine: (1) it is unaffected by maternal antibodies and therefore can be given at any time after birth; (2) BCG is usually given as a single dose eliciting a long-lasting immunity; (3) it is stable and safe; (4) BCG can be administrated orally; and (5) it is inexpensive to produce when compared to other live vaccines. The extraordinary adjuvant properties of mycobacteria make them an attractive vector for the development of recombinant vaccines (Stover et al., 1991; Bastos et al., 2009). Indeed, antigens from bacteria, parasites, and viruses have been expressed in BCG and tested for this purpose (Bastos et al., 2002; Varaldo et al., 2004; Seixas et al., 2007a; da Silva Ramos et al., 2008). In addition, it has been shown that heterologous antigens expressed by recombinant BCG (rBCG) can elicit both cellular and humoral immune responses (Dennehy \& Williamson, 2005).

Despite the success of heterologous antigen expression, and in some cases protection achieved when using $\mathrm{rBCG}$, in vitro and in vivo instability of the recombinant vaccines has been reported (Medeiros et al., 2002; Michelon et al., 2006). This instability is observed mainly when replicative vectors are used, which are lost during BCG replication in vivo (Edelman et al., 1999). Integrative vectors are more stable (Dellagostin et al., 1993).

\footnotetext{
* Corresponding author. Mailling address: Centro de Biotecnologia, Universidade Federal de Pelotas, CP 354, 96010-900, Pelotas, Brazil. Phone: +55 533275 7587. Fax: +55 533275 7551. E-mail: odir@ufpel.edu.br
} 
However, their disadvantage is the reduced expression level of heterologous genes compared to that of multicopy plasmids. Thus, optimization of BCG as a vehicle for live recombinant vaccines requires improved strategies for stable antigen expression. We have recently described the construction of new M. bovis BCG expression vectors using an auxotrophic strain of $M$. bovis BCG and gene complementation as a selectable marker. Preliminary results indicate that this selectable system can be used to express foreign antigens in $\mathrm{BCG}$, maintaining the selective pressure in vivo (Borsuk et al., 2007).

In this report we evaluated the stability of the auxotrophic selection system, comparing it to the conventional system where antibiotic resistance is used as a selective marker. Leptospira interrogans antigens were used for the evaluation of expression stability. The animal species used was the Golden Syrian hamster, a model for animal leptospirosis. Stability was only observed with the new auxotroph complementation system.

\section{MATERIAL AND METHODS}

A BCG auxotrophic for the amino acid leucine was constructed by knocking out the leu $D$ gene by unmarked homologous recombination as previously described (Borsuk et al., 2007). Expression of leuD on a plasmid (pUP410) not only allowed complementation, but also acted as a selectable marker (Borsuk et al., 2007).

To assess functional stability of the leuD complementation system in vivo, the coding sequences for the L. interrogans antigens LipL32 and the non identical region of the LigA antigen (LigAni), together with the 18 $\mathrm{kDa}$ gene promoter from M. Leprae, were amplified by PCR as expression cassettes from pUS2000/lipL32 and pUS2000/ligAni vectors (Seixas et al., 2007b), and cloned into pUP410, an E. coli-mycobacteria shuttle vector that carries the leuD gene, which allows auxotrophic complementation of the BCG $\Delta l e u D$ strain (Borsuk et al., 2007). PCR products were ligated into the
KpnI site of the pUP410 vector, replacing the hygR gene. The resulting vectors were named pUP410+lipL32/18kDa and pUP410+ligAni/18kDa.

Competent mycobacterium cells were prepared as follows: $M$. bovis BCG Pasteur $\Delta l e u \mathrm{D}$ and $M$. bovis $\mathrm{BCG}$ Pasteur were grown in $5 \mathrm{ml}$ to an optical density at 600 $\mathrm{nm}\left(\mathrm{OD}_{600}\right)$ between 0.8 and 1.0 , diluted 100 -fold in $100 \mathrm{ml}$ of the same growth medium and incubated at $37^{\circ} \mathrm{C}$ for 7 days in a shaker. To make competent cells, the cultures were incubated on ice for $1 \mathrm{~h}$, harvested by centrifugation at 4,000 $\mathrm{x} g$ for $10 \mathrm{~min}$ at $4{ }^{\circ} \mathrm{C}$, washed three times with $10 \%$ glycerol $\left(4{ }^{\circ} \mathrm{C}\right)$ and resuspended in 1 $\mathrm{ml}$ of $10 \%$ glycerol. Electroporation was performed following standard methods (Parish \& Stoker, 1998). Briefly, $100 \mu \mathrm{l}$ electrocompetent cells were mixed with 0.5 -1 $\mu \mathrm{g}$ plasmid DNA and electroporated in $0.2 \mathrm{~cm}$ gap electroporation cuvettes at $2.5 \mathrm{kV}, 25 \mu \mathrm{F}$ and $800 \Omega$ using a Gene Pulser II (Bio-Rad). Immediately after electroporation, the cells were diluted in 1 $\mathrm{ml}$ of $7 \mathrm{H} 9$ broth and incubated at $37{ }^{\circ} \mathrm{C}$ for $24 \mathrm{~h}$ (M. bovis BCG) before plating on $7 \mathrm{H} 10$ agar containing the appropriate antibiotic. Transformants were selected after 21 days of incubation at $37{ }^{\circ} \mathrm{C}$. The expression of LipL32 and LigAni in BCG was demonstrated by Western blot (WB), as previously described (Seixas et al., 2007b).

Four-to-six-week-old hamsters were used to evaluate the in vivo stability of the rBCG. In the experiment, forty-eight animals were randomly allocated into four groups, twelve animals per group, and inoculated with approximately $10^{6} \mathrm{CFU}$ of rBCG in $100 \mu$ l of sterile PBS-T. Group one was inoculated with rBCG $\Delta$ leuD (pUP410+lipL32), group two with rBCG $\Delta l e u D$ (pUP410), group three with $\mathrm{rBCG}$ Pasteur (pUP402+lipL32), and group four with rBCG Pasteur (pUP410). At weeks 2, $5,8,12,16$ and 18 after inoculation two animals per group were euthanized and the spleen was removed, homogenized, serially diluted in $7 \mathrm{H} 9$, and plated onto $7 \mathrm{H} 10$ with or without selection (L-leucine for $\mathrm{rBCG}$ $\triangle l e u D$, and kanamycin for $\mathrm{rBCG}$ Pasteur). The number of resulting colonies was compared. Stability was calculated by 
comparing the number of colonies on nonselective medium in relation to the number of colonies on selective medium.

Antibody responses were monitored by indirect ELISA using purified rLipL32 or rLigAni. Polystyrene plates were coated overnight with $500 \mathrm{ng}$ of rLipL32 or rLigAni per well, diluted in carbonate-bicarbonate buffer $\mathrm{pH}$ 9.6, washed three times and sera were diluted in 1: 50 PBS-T. After incubation for $1 \mathrm{~h}$ at $37{ }^{\circ} \mathrm{C}$, followed by three washes with PBS-T, goat anti-hamster IgG peroxidase conjugate (Serotec, USA) was added and the reaction visualized with o-phenylenediamine dihydrochloride (Sigma) and hydrogen peroxide. Absorbance was determined at $450 \mathrm{~nm}$ in Multiskan MCC/340 (Titertek Instruments, USA) ELISA reader. Mean values were calculated from serum samples assayed in duplicate.

\section{RESULTS AND DISCUSSION}

Auxotrophic BCG strains, when inside macrophages, are unable to access intracellular amino acids, thus they fail to grow in vivo (Bange et al., 1996). This important observation opened the possibility of using auxotrophic complementation as a selectable marker, an approach that has two main advantages: it provides active selection in vivo, unlike antibiotic resistance markers, and it abolishes the need for using an antibiotic resistance gene as a vector component. The auxotroph complementation system for stable cloning and expression of foreign genes in BCG is based on the use of a $M$. bovis $\mathrm{BCG}$ strain auxotrophic for the leucine amino acid.

The cloning of the lipL32 or ligAni coding sequences, under expression of the $M$. leprae $18 \mathrm{kDa}$ gene promoter, into pUP410 resulted in the vectors named pUP410+lipL32/18kDa and pUP410+ligAnil $18 \mathrm{kDa}$, respectively. These were then used to transform $M$. bovis $B C G$ Pasteur $\triangle l e u D$ and $M$. bovis BCG Pasteur. Expression of LipL32 or LigAni in BCG was evaluated by Western blot using Monoclonal antibodies (MAbs) against LipL32 or LigAni (Fernandes et al., 2007). These MAbs recognized a protein of approximately 30 $\mathrm{kDa}$ in $\mathrm{rBCG}$ cell lysates expressing LipL32 and approximately $60 \mathrm{kDa}$ in $\mathrm{rBCG}$ cell lysates expressing LigAni. In contrast, no band was detected in wild type BCG extract, demonstrating the specificity of these antibodies. Based on band intensities, the level of expression of LipL32 or LigAni shown by recombinant BCG grown in vitro was considered to be similar for all constructs used (Figure 1).

Stability of M. bovis BCG Pasteur $\Delta l e u D$ and $M$. bovis BCG Pasteur transformed with plasmids containing the leuD gene as a selectable marker was evaluated in hamsters. Initial selection was carried out on medium without leucine for BCG $\Delta l e u D$ or containing kanamycin for BCG Pasteur. Approximately $10^{6} \mathrm{CFU}$ of each strain were
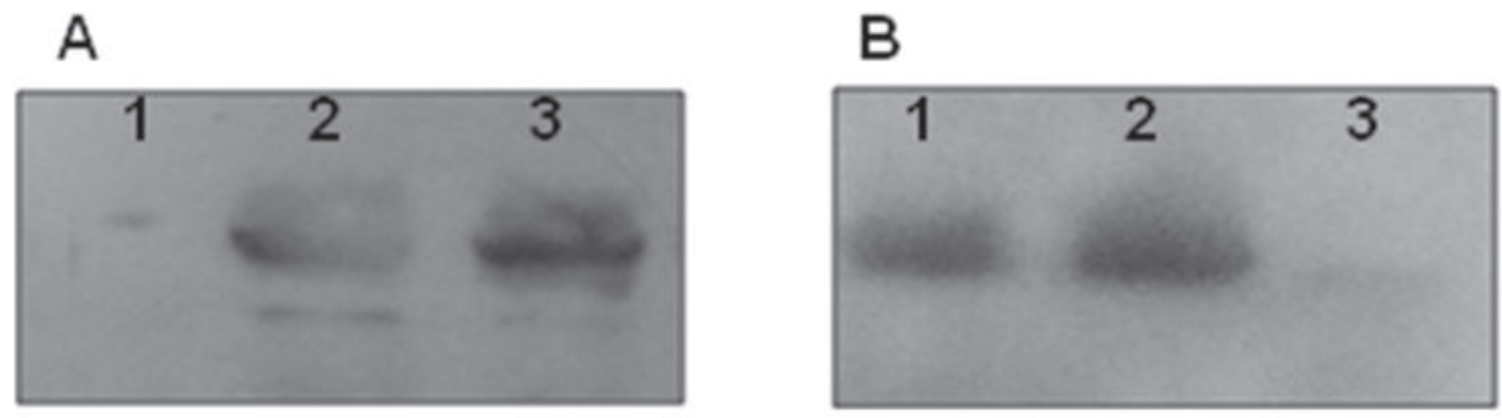

Figure 1: Western blot using monoclonal antibodies (MAbs) against LipL32 or LigAni demonstrating LipL32 and LigAni expressions in rBCG. Panel A- Lane 1, wtBCG (control); lane 2, rBCG $\Delta l e u D$ transformed with pUP410+ligani/18kDa; lane 3, rBCG $\Delta$ leuD transformed with pUP410+ligani/18kDa; Panel B- Lane 1, rBCG $\Delta$ leuD transformed with pUP410+lipL32/18kDa; lane 2, rBCG Pasteur transformed with pUP410+lipL32/18kDa; lane 3, wtBCG (control). 
inoculated into hamsters. Hamsters were inoculated with BCG $\triangle l e u D$ transformed with pUP410+lipL32/18kDa and pUP410+ligani/18kDa and BCG Pasteur transformed with the same vectors. Bacteria cells were recovered from the spleens of two animals killed at 2, 5, 8, 12, 16 and 18 weeks post inoculation and plated on selective and non-selective medium. The ratio of resistant (BCG carrying the plasmid vector) versus total $\mathrm{BCG}$ colonies were calculated for each strain. Stability of BCG $\triangle l e u D$ and BCG Pasteur strains is shown in figure 2. Auxotrophic complementation vectors used to transform BCG $\Delta l e u D$ showed $100 \%$ stability in vivo during the 18 weeks of the experiment (Figure 2). Conversely, the same vectors used to transform BCG Pasteur were lost during in vivo growth. At two weeks post-inoculation less than $50 \%$ of the bacterial cells still harboured the plasmid. This number fell to $7 \%$ at 18 weeks post-inoculation (Figure 2). Interestingly, it was noted that there was no significant reduction in the number of CFU recovered from hamsters inoculated with $\mathrm{BCG} \Delta$ leuD complemented strains in comparison to rBCG Pasteur. A difference would be expected as BCG $\Delta l e u D$ cells that lose the plasmid fail to survive due to the unavailability of exogenous leucine inside macrophage cells. In a similar experiment performed in mice, a high level of functional stability was also observed as all the recovered BCG $\Delta l e u D$ transformed with vectors carrying lac $\mathrm{Z}$ gave rise to blue colonies when plated onto medium containing X-gal (Borsuk et al., 2007).

The humoral immune response against both leptospiral recombinant antigens induced by rBCG Pasteur $\Delta l e u D$ and rBCG Pasteur expressing the leptospiral antigens was evaluated by ELISA at 42 days after vaccination. No difference between the two strains could be observed at the evaluated point in time. The reason for that is probably that when the vaccine is administered the level of expression of the foreign antigen is high in both strains. The loss of the plasmid vector in the $\mathrm{rBCG}$ Pasteur strain that occurred after inoculation will only result in a lower immune response in the long term.
The use of auxotrophic complementation as a selectable marker for expression of foreign genes in BCG described in this report provides the high levels of stability previously described only for integrative vectors (Mederle et al., 2002). However, it has the advantage of a multicopy vector, namely higher levels of foreign antigen expression. In addition, the stability of the auxotrophic complementation system is of utmost importance for the expression of heterologous antigens. The permanence of the expression vector in $\mathrm{rBCG}$ offers the possibility of continued production of the recombinant antigen, constantly stimulating the immune system. An appropriate immune response against the recombinant antigen may depend on the length of time the immune system is stimulated.

In addition, an antibiotic resistance gene would not be allowed in a live recombinant vaccine. In our study the kanamycin resistance gene was still present, as it was required for selection in the BCG Pasteur strain. However, the pUP410 vector was constructed in such a way that a simple restriction digestion and re-ligation are sufficient for removing the kanamycin resistance gene prior to transformation of BCG $\Delta l e u D$ strain (Borsuk et al., 2007). This vector can be used to clone and express virtually any foreign gene in BCG.

Confirmation of rBCG stability during in vitro growth using the auxotrophic complementation system as selectable marker had previously been demonstrated only in mice (Borsuk et al., 2007). This is the first report of evaluation of $\mathrm{rBCG}$ stability in hamster. Recombinant BCG expressing LipL32 antigen from $L$. interrogans has been shown to protect hamsters from a lethal challenge, using a conventional expression system (Seixas et al., 2007a). We are confident that the improved stability provided by the auxotrophic complementation system will be able to further enhance the immune response elicited by $\mathrm{rBCG}$ expressing LipL32, conferring a higher protection level. Experiments are currently been conducted to test this hypothesis. If confirmed, other rBCG vaccine candidates that have already been demonstrated to 


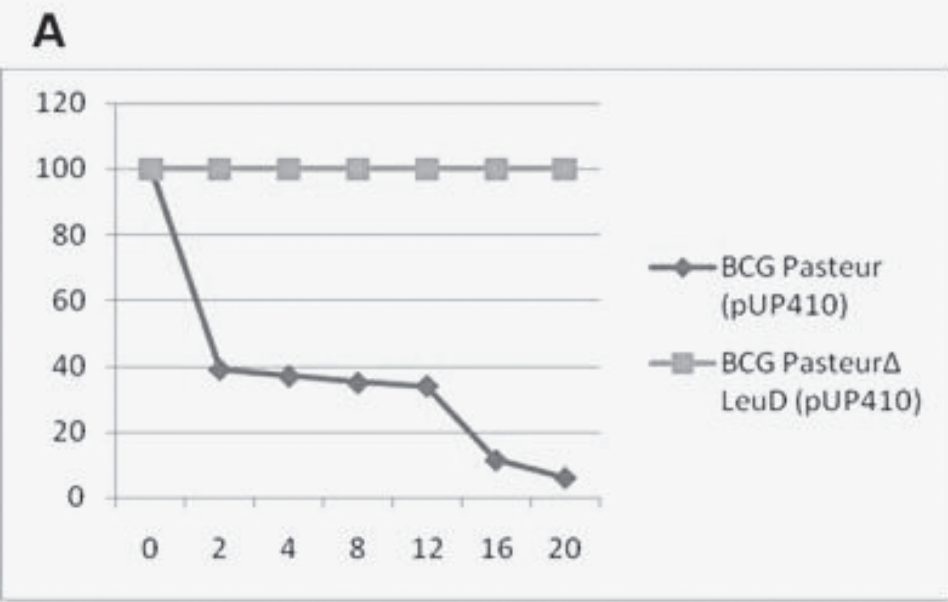

B

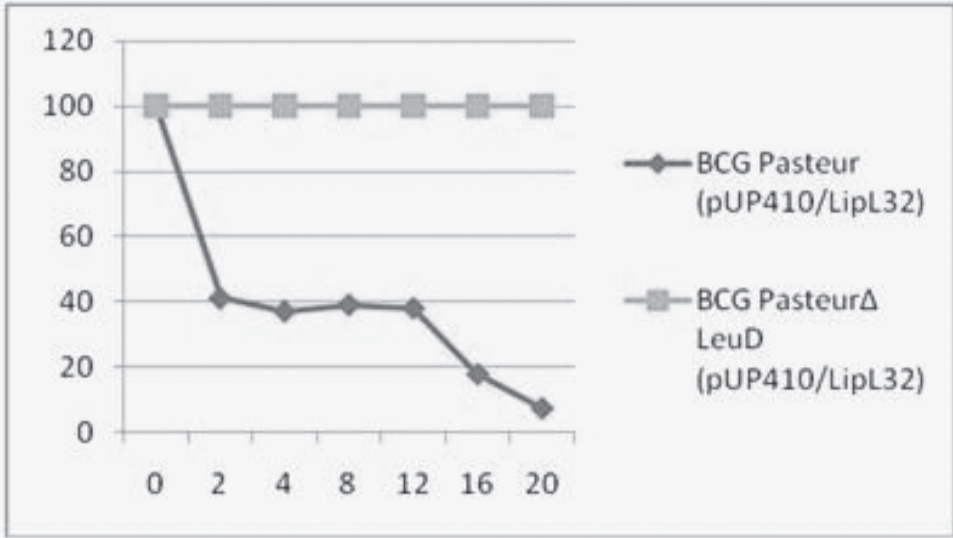

\section{C}

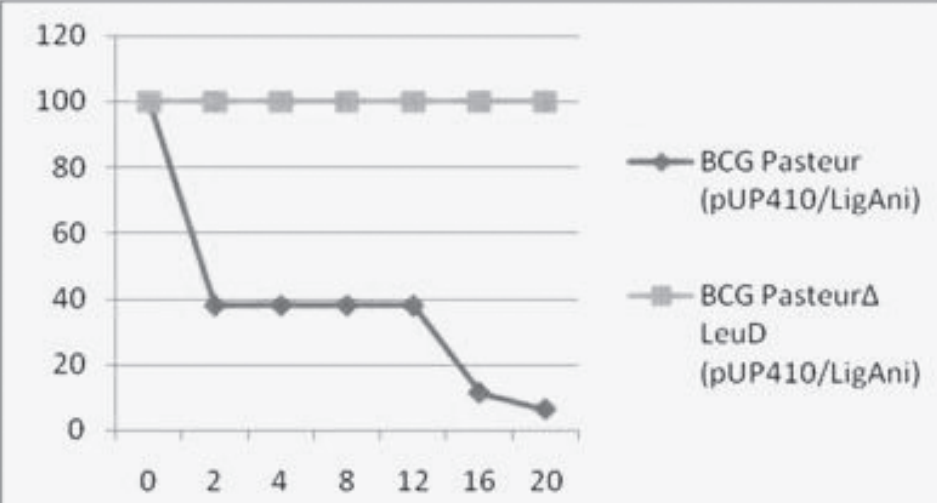

Figure 2: In vivo stability of $\mathrm{rBCG}$ transformed with vectors containing auxotrophic complementation. Hamsters were inoculated with BCG $\Delta$ leuD transformed with pUP410, pUP410+lipL32/18kDa and pUP410+ligani/18kDa and BCG Pasteur transformed with the same vectors. Bacteria were recovered from spleen of three animals killed at 2, 4, 8, 12, 16 and 20 weeks post inoculation and plated on selective and non-selective medium. Ratios of resistant (rBCG) versus total BCG colonies were calculated for each strain and construction tested (A, B and C). 
induce protection in animal model experiments (Connell et al., 1993; Buddle et al., 1995; Abomoelak et al., 1999; Demangel et al., 2005; Dennehy et al., 2007; Seixas et al., 2007a) could also be further improved by the use of the new expression system.

\section{REFERENCES}

ABOMOELAK B, HUYGEN K, KREMER L, TURNEER M, \& LOCHT C (1999). Humoral and cellular immune responses in mice immunized with recombinant Mycobacterium bovis Bacillus Calmette-Guerin producing a pertussis toxin-tetanus toxin hybrid protein. Infect Immun 67: 5100-5105.

BANGE FC, BROWN AM, \& JACOBS WR, JR. (1996). Leucine auxotrophy restricts growth of Mycobacterium bovis BCG in macrophages. Infect Immun 64: 17941799.

BASTOS RG, BORSUK S, SEIXAS FK, \& DELLAGOSTIN OA (2009). Recombinant Mycobacterium bovis BCG. Vaccine 27: 6495-6503.

BASTOS RG, DELLAGOSTIN OA, BARLETTA RG, DOSTER AR, NELSON E, \& OSORIO FA (2002). Construction and immunogenicity of recombinant Mycobacterium bovis BCG expressing GP5 and M protein of porcine reproductive respiratory syndrome virus. Vaccine 21: 21-29.

BORSUK S, MENDUM TA, FAGUNDES MQ, MICHELON M, CUNHA CW, MCFADDEN J, \& DELlagostiN OA (2007). Auxotrophic complementation as a selectable marker for stable expression of foreign antigens in Mycobacterium bovis BCG. Tuberculosis 87: 474-480.

BUDDLE BM, DE LISLE GW, PFEFFER A, \& ALDWELL FE (1995). Immunological responses and protection against Mycobacterium bovis in calves vaccinated with a low dose of BCG. Vaccine 13: 1123-1130.

CONNELL ND, MEDINA-ACOSTA E, MCMASTER WR, BLOOM BR, \& RUSSELL DG (1993). Effective immunization against cutaneous leishmaniasis with recombinant bacille Calmette-Guerin expressing the Leishmania surface proteinase gp63. Proc Natl Acad Sci U S A 90: 11473-11477.

DA SILVA RAMOS RA, CONCEICAO FR, GRASSMANN AA, LAGRANHA VL, \& DELLAGOSTIN OA (2008). B subunit of Escherichia coli heat-labile enterotoxin as adjuvant of humoral immune response in recombinant BCG vaccination. Can J Microbiol 54: 677-686.

DELLAGOSTIN OA, WALL S, NORMAN E, O'SHAUGHNESSY T, DALE JW, \& MCFADDEN J (1993). Construction and use of integrative vectors to express foreign genes in mycobacteria. Mol Microbiol 10: 983-993.

DEMANGEL C, GARNIER T, ROSENKRANDS I, \& COLE ST (2005). Differential effects of prior exposure to environmental mycobacteria on vaccination with Mycobacterium bovis $\mathrm{BCG}$ or a recombinant $\mathrm{BCG}$ strain expressing RD1 antigens. Infect Immun 73: 2190-2196.

DENNEHY M, BOURN W, STEELE D, \& WILLIAMSON AL (2007). Evaluation of recombinant BCG expressing rotavirus VP6 as an anti-rotavirus vaccine. Vaccine 25: 3646-3657.

DENNEHY M \& WILLIAMSON AL (2005). Factors influencing the immune response to foreign antigen expressed in recombinant BCG vaccines. Vaccine 23: 1209-1224.

EDELMAN R, PALMER K, RUSS KG, SECREST HP, BECKER JA, BODISON SA, PERRY JG, SILLS AR, BARBOUR AG, LUKE CJ, HANSON MS, STOVER CK, BURLEIN JE, BANSAL GP, CONNOR EM, \& KOENIG S (1999). Safety and immunogenicity of recombinant Bacille Calmette-Guerin (rBCG) expressing Borrelia burgdorferi outer surface protein A (OspA) lipoprotein in adult volunteers: a candidate Lyme disease vaccine. Vaccine 17: 904-914.

FERNANDES CP, SEIXAS FK, COUTINHO ML, VASCONCELLOS FA, SEYFFERT N, CRODA J, MCBRIDE AJ, KO AI, DELLAGOSTIN OA, \& ALEIXO JA (2007). Monoclonal antibodies against LipL32, the major outer membrane protein of pathogenic Leptospira: production, characterization, and testing in diagnostic applications. Hybridoma 26: $35-41$.

MEDEIROS MA, DELLAGOSTIN OA, ARMOA GR, DEGRAVE WM, MENDONCA-LIMA L, LOPES MQ, COSTA JF, MCFADDEN J, \& MCINTOSH D (2002). Comparative evaluation of Mycobacterium vaccae as a surrogate cloning host for use in the study of mycobacterial genetics. Microbiology 148: 1999-2009.

MEDERLE I, BOURGUIN I, ENSERGUEIX D, BADELL E, MONIZ-PEIREIRA J, GICQUEL B, \& WINTER N (2002). Plasmidic versus insertional cloning of heterologous genes in Mycobacterium bovis BCG: impact on in vivo antigen persistence and immune responses. Infect Immun 70: 303-314.

MICHELON A, CONCEIÇÃO FR, BINSFELD PC, DA CUNHA CW, MOREIRA AN, ARGONDIZZO AP, MCINTOSH D, ARMOA GR, CAMPOS AS, FARBER M, MCFADDEN J, \& DELLAGOSTIN OA (2006). Immunogenicity of Mycobacterium bovis BCG expressing Anaplasma marginale MSP1a antigen. Vaccine 24: 6332-6339.

PARISH T \& STOKER NG (1998). Electroporation of mycobacteria. Methods Mol Biol 101: 129-144.

SEIXAS FK, DA SILVA EF, HARTWIG DD, CERQUEIRA GM, AMARAL M, FAGUNDES MQ, DOSSA RG, \& DELLAGOSTIN OA (2007a). Recombinant Mycobacterium bovis BCG expressing the LipL32 antigen of Leptospira interrogans protects hamsters from challenge. Vaccine 26: 88-95.

SEIXAS FK, FERNANDES CH, HARTWIG DD, CONCEIÇÃO FR, ALEIXO JA, \& DELLAGOSTIN OA (2007b). Evaluation of different ways of presenting LipL32 to the immune system with the aim of developing a recombinant vaccine against leptospirosis. Can J Microbiol 53: 472-479.

STOVER CK, DE LC, V, FUERST TR, BURLEIN JE, BENSON LA, BENNETT LT, BANSAL GP, YOUNG JF, LEE MH, HATFULL GF. (1991). New use of BCG for recombinant vaccines. Nature 351: 456-460.

VARALDO PB, LEITE LC, DIAS WO, MIYAJI EN, TORRES FI, GEBARA VC, ARMOA GR, CAMPOS AS, MATOS DC, WINTER N, GICQUEL B, VILAR MM, MCFADDEN J, ALMEIDA MS, TENDLER M, \& MCINTOSH D (2004). Recombinant Mycobacterium bovis BCG expressing the Sm14 antigen of Schistosoma mansoni protects mice from cercarial challenge. Infect Immun 72: 3336-3343. 